\title{
A VARIATIONAL METHOD FOR UNIVALENT FUNCTIONS CONNECTED WITH ANTIGRAPHY
}

\author{
JANINA MACURA \\ Institute of Mathematics, Silesian Technical University \\ Kaszubska 23, PL-44-100 Gliwice, Poland
}

\begin{abstract}
The paper is devoted to a class of functions analytic and univalent in the unit disk that are connected with an antigraphy $e^{i \varphi} \bar{w}+i \rho e^{i \frac{\varphi}{2}}$. Variational formulas and Grunsky inequalities are derived. As an application there are given some estimations in the considered class of functions.
\end{abstract}

1. Introduction. $H(U)$ denotes, as usual, the space of all functions analytic in the unit disk $U=\{z:|z|<1\}$. Let $\rho \in \mathbb{R}, \varphi \in[0,2 \pi], a \in \mathbb{C}$, and $\rho \neq 2 \operatorname{Im}\left\{e^{-i \frac{\varphi}{2}} a\right\}$. $S_{a \rho \varphi}$ denotes the class of all functions that are analytic, univalent in the unit disk $U$ and satisfy the conditions

$$
f(0)=a \quad \text { and } \quad f\left(z_{1}\right) \neq e^{i \varphi} \overline{f\left(z_{2}\right)}+i \rho e^{i \frac{\varphi}{2}}, \quad z_{1}, z_{2} \in U .
$$

The class $S_{a \rho \varphi}$ is, in some sense, similar to the classes of Gel'fer, Bieberbach-Eilenberg, Grunsky-Shah and bounded functions. We can write the definitions of these classes in a common form as follows:

Let $J$ be a class of all functions that are analytic and univalent in $U$ and satisfy the conditions

$$
f(0)=a \quad \text { and } \quad w \in f(U) \Longrightarrow \omega(w) \notin f(U) .
$$

For $a=1$ and $\omega(w)=-w J$ is the class of Gel'fer functions, for $a=0$ and $\omega(w)=\frac{1}{w}$ - the class of Bieberbach-Eilenberg functions, for $a=0$ and $\omega(w)=-\frac{1}{w}-$ the class of Grunsky-Shah functions, for $a=0$ and $\omega(w)=\frac{1}{w}$ - the class of bounded functions, and finally for $\omega(w)=e^{i \varphi} \bar{w}+i \rho e^{i \frac{\varphi}{2}}-$ the class $S_{a \rho \varphi}$. Each of these homographies and antigraphies has the property that the inverse function is the same.

The class $S_{10 \pi}$ coincides with the class of univalent functions with positive real part.

2. Variational formulas. Let $f \in S_{a \rho \varphi}$ and $D=f(U)$. It is clear that the domain

1991 Mathematics Subject Classification: Primary 30 C 70; Secondary 30 C45.

The paper is in final form and no version of it will be published elsewhere. 
$D$ has the property

$$
w \in D \Longrightarrow e^{i \varphi} \bar{w}+i \rho e^{i \frac{\varphi}{2}} \notin D .
$$

Using the Golusin's method we can derive the variational formula for the function $f$.

TheOREM 1. Let $f \in S_{a \rho \varphi}, z_{0} \in U$. Then for sufficiently small $\varepsilon$ there exists a function $f^{*} \in S_{a \rho \varphi}$ such that

(3) $f^{*}(z)=f(z)+\varepsilon\left\{e^{i \alpha}\left[\frac{(f(z)-a)(f(z)-b)}{f(z)-f\left(z_{0}\right)}-\frac{\left(f\left(z_{0}\right)-a\right)\left(f\left(z_{0}\right)-b\right)}{z_{0} f^{\prime 2}\left(z_{0}\right)} \frac{z f^{\prime}(z)}{z-z_{0}}\right]+\right.$

$$
\left.+e^{-i \alpha}\left[\frac{(f(z)-a)(f(z)-b)}{f(z)-e^{i \varphi} \overline{f\left(z_{0}\right)}-i \rho e^{i \frac{\varphi}{2}}}+\frac{\overline{\left(f\left(z_{0}\right)-a\right)\left(f\left(z_{0}\right)-b\right)}}{\overline{z_{0} f^{\prime 2}\left(z_{0}\right)}} \frac{z^{2} f^{\prime}(z)}{1-\overline{z_{0} z}}\right]\right\}+o(\varepsilon)
$$

where $\frac{o(\varepsilon)}{\varepsilon} \rightarrow 0$, while $\varepsilon \rightarrow 0$, uniformly on compact subsets of $U$.

P r o of. In order to find the variation of the function $f$ we shall at first define such a variation $w^{*}$ of the boundary $\partial D$ that does not violate the property (2) for the domain $D^{*}$ bounded by $w^{*}(\partial D)$. Define the function

$$
w^{*}(w)=w+\varepsilon v(w),
$$

where $\varepsilon>0, v(w)$ is a function analytic in the closure of such a domain $\Delta$ that contains $\partial D$ and satisfies the condition

$$
w \in \Delta \Longrightarrow e^{i \varphi} \bar{w}+i \rho e^{i \frac{\varphi}{2}} \in \Delta
$$

and having the property

$$
v\left(e^{i \varphi} \bar{w}+i \rho e^{i \frac{\varphi}{2}}\right)=e^{i \varphi} \overline{v(w)} .
$$

Following [3] one can prove that the function (4) is univalent for sufficiently small $\varepsilon$ and $w^{*}(\partial D)$ is a boundary of a domain $D^{*}$ having the property $(2)$.

Let $P=\{z: r \leq|z|<1\}, r \in(0,1)$ be such a ring that $f(P) \subset \Delta$. The function

$$
F(z, \varepsilon)=w^{*}(f(z))-a, \quad z \in P
$$

satisfies the assumptions of Golusin theorem [2] for the function $f(z)-a$. So the function $f^{*}$ such that $f^{*}(U)=D^{*}$ and $f^{*}(0)=a$ has the form

$$
f^{*}(z)=f(z)+\varepsilon\left\{v(f(z))-z f^{\prime}(z) S(z)+z f^{\prime}(z) \overline{S\left(\frac{1}{\bar{z}}\right)}\right\}+o(\varepsilon),
$$

where $S(z)$ is a principal part of the development into a Laurent series of the function $\frac{v(f(z))}{z f^{\prime}(z)}$ and $\frac{o(\varepsilon)}{\varepsilon} \rightarrow 0$, while $\varepsilon \rightarrow 0$, uniformly on compact subsets of $U$. The function $f^{*}$ belongs to the class $S_{a \rho \varphi}$ and is a variation of the function $f$.

Now, we define the function $v(w)$ as follows

$$
v(w)=(w-a)(w-b)\left(\frac{e^{i \alpha}}{w-w_{0}}+\frac{e^{-i \alpha}}{w-e^{i \varphi} \overline{w_{0}}-i \rho e^{i \frac{\varphi}{2}}}\right),
$$

where $w_{0}=f\left(z_{0}\right), z_{0} \in U, b=e^{i \varphi} \bar{a}+i \rho e^{i \frac{\varphi}{2}}, \alpha$ is an arbitrary real number. It is clear that $v(w)$ satisfies the condition (5). The variation (6) in this case takes the form (3). 
We can also obtain other variational formulas. If $w_{0} \notin \bar{D}$ and $e^{i \varphi} \overline{w_{0}}+i \rho e^{i \frac{\varphi}{2}} \notin \bar{D}$ then we have

$$
\begin{gathered}
f^{*}(z)=f(z)+\varepsilon\left\{e^{i \alpha} \frac{(f(z)-a)(f(z)-b)}{f(z)-w_{0}}+\right. \\
\left.+e^{-i \alpha} \frac{(f(z)-a)(f(z)-b)}{f(z)-e^{i \varphi} \overline{w_{0}}-i \rho e^{i \frac{\varphi}{2}}}\right\}+o(\varepsilon),
\end{gathered}
$$

where $\frac{o(\varepsilon)}{\varepsilon} \rightarrow 0$, while $\varepsilon \rightarrow 0$, uniformly on compact subsets of $U$.

Compositions of a function $f \in S_{a \rho \varphi}$ with univalent functions $g$ such that $g(0)=0$ and $g(U) \subset U$ give other variations of $f$, for example:

$$
f^{*}(z)=f\left(e^{i \varepsilon} z\right)=f(z)+i \varepsilon z f^{\prime}(z)+o(\varepsilon), \quad \varepsilon \in \mathbb{R}
$$

and

$$
f^{*}(z)=f\left(k_{\alpha}^{-1}\left((1-\varepsilon) k_{\alpha}(z)\right)\right)=f(z)-\varepsilon z f^{\prime}(z) \frac{e^{i \alpha}+z}{e^{i \alpha}-z}+o(\varepsilon),
$$

where $k_{\alpha}(z)=\frac{z}{\left(1+e^{-i \alpha} z\right)^{2}}, \alpha \in \mathbb{R}, \varepsilon>0$, and where $\frac{o(\varepsilon)}{\varepsilon} \rightarrow 0$, while $\varepsilon \rightarrow 0$, uniformly on compact subsets of $U$.

3. Schiffer equation. $S_{a \rho \varphi}$ is a normal family of functions. It becomes compact if we add the constant function $g=a$. The family of functions close to the function $f \in S_{a \rho \varphi}$ that we have just constructed is rich enough to consider the maximal problem in the class $S_{a \rho \varphi}$. Let $\psi$ be a complex, continuous functional defined over $S_{a \rho \varphi}$. Suppose that $\operatorname{Re}\{\psi\}$ has a Fréchet derivative at the point $f \in S_{a \rho \varphi}$. Then there exists a functional $L_{f} \in H^{\prime}(U)$ such that

$$
\operatorname{Re}\left\{\psi\left(f^{*}\right)\right\}=\operatorname{Re}\{\psi(f)\}+\varepsilon \operatorname{Re}\left\{L_{f}(h)\right\}+o(\varepsilon),
$$

for every function

$$
f^{*}(z)=f(z)+\varepsilon h(z)+o(\varepsilon)
$$

such that $h \in H(U), \frac{o(\varepsilon)}{\varepsilon} \rightarrow 0$, while $\varepsilon \rightarrow 0$, uniformly on compact subsets of $U$.

TheOREM 2. Let $\psi$ be a complex functional defined and continuous over the class $S_{a \rho \varphi}$ and let $\operatorname{Re}\{\psi\}$ have a Fréchet derivative $L_{f}$ at the point $f \in S_{a \rho \varphi}$. If $\operatorname{Re}\{\psi\}$ attains its maximal value in the class $S_{a \rho \varphi}$ at $f$ then $f$ satisfies the equation

$$
\frac{\left(\zeta f^{\prime}(\zeta)\right)^{2}}{(f(\zeta)-a)(f(\zeta)-b)} A(f(\zeta))=B(\zeta),
$$

where $A(w)$ and $B(z)$ are given by the formulas:

$$
\begin{gathered}
A(w)=L_{f}\left(\frac{(f(z)-a)(f(z)-b)}{f(z)-w}\right)+\overline{L_{f}\left(\frac{(f(z)-a)(f(z)-b)}{f(z)-e^{i \varphi} \bar{w}-i \rho e^{i \frac{\varphi}{2}}}\right)}, \\
B(\zeta)=L_{f}\left(\frac{\zeta z f^{\prime}(z)}{z-\zeta}\right)+\overline{L_{f}\left(z f^{\prime}(z)\right)}-\overline{L_{f}\left(\frac{z f^{\prime}(z)}{1-\bar{\zeta} z}\right)},
\end{gathered}
$$

$r<|\zeta|<1, r \in(0,1)$. The function $B(\zeta)$ is an analytic function in the ring $P_{r}=\{\zeta$ : $\left.r<|\zeta|<\frac{1}{r}\right\}$, is real and non-positive on $\partial U$. 
Proof. If the functional $\operatorname{Re}\{\psi\}$ attains at $f \in S_{a \rho \varphi}$ its maximal value and $f^{*}$ has the form (3) then (10) leads to

$$
\frac{\left(z_{0} f^{\prime}\left(z_{0}\right)\right)^{2}}{\left(f\left(z_{0}\right)-a\right)\left(f\left(z_{0}\right)-b\right)} A\left(f\left(z_{0}\right)\right)=B\left(z_{0}\right),
$$

where $A(w)$ and $B(\zeta)$ are given by the formulas (12). Combining (8) with (10) and (9) with (10) and using the fact that $f$ is maximal we conclude that $B(\zeta)$ is real and non-positive on $\partial U$, which completes the proof.

As a consequence of applying the variational formula (7) to (10) we have the following theorem:

THEOREM 3. Let $\psi$ and $f$ satisfy the assumptions of the previous theorem, $A$ be such a function meromorphic in $\mathbb{C}$ that $A \neq 0$. If $w_{0}$ and $e^{i \varphi} \overline{w_{0}}+i \rho e^{i \frac{\varphi}{2}}$ are not in $f(U)$ then at least one of these points is on the boundary $\partial f(U)$. Particularly the set $\mathbb{C}-(f(U) \cup h(U))$, where $h(z)=e^{i \varphi} \overline{f(z)}+i \rho e^{i \frac{\varphi}{2}}$ has no interior points.

4. Grunsky inequalities. Defining the functional $\psi$ in a special way we can obtain the complete square on the left-hand side of (11) and then find a solution of this equation in an implicit form. Such a functional leads also to Grunsky inequalities and then to some simple estimations in the class $S_{a \rho \varphi}$. Let

$$
\begin{gathered}
\psi(f)=\lambda^{2} \log \frac{f^{\prime}(0)}{a-b}+2 \lambda L\left(\log \frac{f(z)-a}{z(f(z)-b)}\right)+ \\
+L^{2}\left(\log \frac{f(z)-f(\zeta)}{z-\zeta}\right)-|L|^{2}\left(\log \left(f(z)-e^{i \varphi} \overline{f(\zeta)}-i \rho e^{i \frac{\varphi}{2}}\right)\right),
\end{gathered}
$$

where $L$ is a functional from $H^{\prime}(U)$ such that $L(1)=0, L^{2}(\varphi(z, \zeta))=L(L(\varphi(z, \zeta))),|L|^{2}(\varphi(z, \bar{\zeta}))=L(\overline{L(\varphi(z, \bar{\zeta}))})$ for $\varphi(z, \zeta)$ analytic in $U \times U, \lambda$ is an arbitrary real number.

The Fréchet derivative of $\operatorname{Re}\{\psi\}$ exists for every $f \in S_{a \rho \varphi}$ and has the form

$$
\begin{aligned}
\operatorname{Re}\left\{L_{f}(h)\right\}=\operatorname{Re}\left\{\lambda^{2} \frac{h^{\prime}(0)}{f^{\prime}(0)}+2 \lambda L\left(\frac{(a-b) h(z)}{(f(z)-a)(f(z)-b)}\right)+L^{2}\left(\frac{h(z)-h(\zeta)}{f(z)-f(\zeta)}\right)-\right. \\
\left.-|L|^{2}\left(\frac{h(z)}{f(z)-e^{i \varphi} \overline{f(\zeta)}-i \rho e^{i \frac{\varphi}{2}}}\right)+|L|^{2}\left(\frac{e^{i \varphi} \overline{h(\zeta)}}{f(z)-e^{i \varphi} \overline{f(\zeta)}-i \rho e^{i \frac{\varphi}{2}}}\right)\right\} .
\end{aligned}
$$

THEOREM 4. If the functional (13) attains its maximal value at the point $f \in S_{a \rho \varphi}$ then $f$ satisfies the equation

$$
\text { (15) } \begin{aligned}
\lambda & \log \frac{f(\zeta)-a}{\zeta(f(z)-a)}+L\left(\log \frac{f(z)-f(\zeta)}{z-\zeta}\right)-\overline{L\left(\log \left(f(z)-e^{i \varphi} \overline{f(\zeta)}-i \rho e^{i \frac{\varphi}{2}}\right)\right)}+ \\
& +\overline{L(\log (1-\bar{\zeta} z))}=\lambda \log \frac{f^{\prime}(0)}{a-b}+L\left(\log \frac{f(z)-a}{z}\right)-\overline{L(\log (f(z)-b))} .
\end{aligned}
$$

The maximal value $\operatorname{Re}\{\psi(f)\}=-|L|^{2}(\log (1-\bar{\zeta} z))$.

Proof. Let $f \in S_{a \rho \varphi}$ be a maximal function for the functional $\operatorname{Re}\{\psi\}$. According to the theorem 2 the function $f$ satisfies the equation (11). In our case this equation has 
the form

$$
\begin{gathered}
\left(\zeta f^{\prime}(\zeta)\right)^{2}\left(\lambda \frac{a-b}{(f(\zeta)-a)(f(\zeta)-b)}-L\left(\frac{1}{f(z)-f(\zeta)}\right)+\right. \\
\left.+e^{-i \varphi} L\left(\frac{1}{f(z)-e^{i \varphi} \overline{f(\zeta)}-i \rho e^{i \frac{\varphi}{2}}}\right)\right)^{2}=-B(\zeta) .
\end{gathered}
$$

From the Caccioppoli-Kőthe integral representation of the functional from $H^{\prime}(U)$ [1] and from the fact that $B(\zeta)$ is non-positive on $\partial U$ and from (16), following [4], we conclude that the function

$$
\begin{aligned}
C(\zeta)=\lambda & \frac{(a-b) \zeta f^{\prime}(\zeta)}{(f(\zeta)-a)(f(\zeta)-b)}-L\left(\frac{\zeta f^{\prime}(\zeta)}{f(z)-f(\zeta)}-\frac{\zeta}{z-\zeta}\right)+ \\
& +L\left(\frac{e^{i \varphi} \overline{\zeta f^{\prime}(\zeta)}}{f(z)-e^{i \varphi} \overline{f(\zeta)}-i \rho e^{i \frac{\varphi}{2}}}-\frac{1}{1-\bar{\zeta} z}\right)
\end{aligned}
$$

is analytic in $U$ and has such a continuous continuation tu $\bar{U}$ that is real on $\partial U$. Furthermore, we notice that it is constant and this constant is equal to $\lambda$ and we have

$$
\begin{aligned}
& \zeta f^{\prime}(\zeta)\left(\lambda \frac{a-b}{(f(\zeta)-a)(f(\zeta)-b)}-L\left(\frac{1}{f(z)-f(\zeta)}\right)+\right. \\
& \left.+e^{-i \varphi} L\left(\frac{1}{f(z)-e^{i \varphi} \overline{f(\zeta)}-i \rho e^{i \frac{\varphi}{2}}}\right)\right)=\lambda-L\left(\frac{\zeta}{z-\zeta}\right)+\overline{L\left(\frac{1}{1-\bar{\zeta} z}\right)} .
\end{aligned}
$$

Now it is easy to verify that

$$
\begin{gathered}
\frac{(a-b) \zeta f^{\prime}(\zeta)}{(f(\zeta)-a)(f(\zeta)-b)}=\zeta \frac{\partial}{\partial \zeta} \log \frac{f(\zeta)-a}{\zeta(f(\zeta)-b)}, \\
\frac{\zeta f^{\prime}(\zeta)}{f(z)-f(\zeta)}-\frac{\zeta}{z-\zeta}=-\zeta \frac{\partial}{\partial \zeta} \log \frac{f(z)-f(\zeta)}{z-\zeta}, \\
\frac{e^{i \varphi} \overline{\zeta f^{\prime}(\zeta)}}{f(z)-e^{i \varphi} \overline{f(\zeta)}-i \rho e^{i \frac{\varphi}{2}}}=-\bar{\zeta} \frac{\partial}{\partial \bar{\zeta}} \log \left(f(z)-e^{i \varphi} \overline{f(\zeta)}-i \rho e^{i \frac{\varphi}{2}}\right), \\
\frac{1}{1-\bar{\zeta} z}=1-\bar{\zeta} \frac{\partial}{\partial \bar{\zeta}} \log (1-\bar{\zeta} z) .
\end{gathered}
$$

Applying (18) to (17) we get

$$
\begin{aligned}
\lambda \log \frac{f(\zeta)-a}{\zeta(f(\zeta)-b)}+L\left(\log \frac{f(z)-f(\zeta)}{z-\zeta}\right)-\overline{L\left(\log \left(f(z)-e^{i \varphi} \overline{f(\zeta)}-i \rho e^{i \frac{\varphi}{2}}\right)\right)}+ \\
+\overline{L(\log (1-\bar{\zeta} z))}=c
\end{aligned}
$$

where

$$
c=\lambda \log \frac{f^{\prime}(0)}{a-b}+L\left(\log \frac{f(z)-a}{z}\right)-\overline{L(\log (f(z)-b))} .
$$

We shall prove that $\operatorname{Re}\{c\}=0$. Notice at first that it follows from the theorem 3 that the boundaries $\partial f(U)$ and $\partial h(U)$ have a common point $\omega$. Then there exist two sequences $\left(\zeta_{n}^{1}\right)$ and $\left(\zeta_{n}^{2}\right)$ of points from $U$ such that $f\left(\zeta_{n}^{1}\right) \rightarrow \omega$ and $h\left(\zeta_{n}^{2}\right) \rightarrow \omega$. Putting correspondingly 
$\zeta_{n}^{1}$ and $\zeta_{n}^{2}$ into (19) and passing to the limit we conclude that $\operatorname{Re}\{c\}=0$ that is

$$
\operatorname{Re}\left\{\lambda \log \frac{f^{\prime}(0)}{a-b}+L\left(\log \frac{f(z)-a}{z}\right)-\overline{L(\log (f(z)-b))}\right\}=0 .
$$

(19) leads also to another equation

$$
\begin{gathered}
\lambda L\left(\log \frac{f(\zeta)-a}{\zeta(f(\zeta)-b)}\right)+L^{2}\left(\log \frac{f(z)-f(\zeta)}{z-\zeta}\right)- \\
-|L|^{2}\left(\log \left(f(z)-e^{i \varphi} \overline{f(\zeta)}-i \rho e^{i \frac{\varphi}{2}}\right)\right)+|L|^{2}(\log (1-\bar{\zeta} z))=0 .
\end{gathered}
$$

Finally adding (21) and (20) multiplied by $\lambda$, we obtain

$$
\begin{gathered}
\operatorname{Re}\left\{\lambda^{2} \log \frac{f^{\prime}(0)}{a-b}+2 \lambda L\left(\log \frac{f(z)-a}{z(f(z)-b)}\right)+L^{2}\left(\log \frac{f(z)-f(\zeta)}{z-\zeta}\right)-\right. \\
\left.-|L|^{2}\left(\log \left(f(z)-e^{i \varphi} \overline{f(\zeta)}-i \rho e^{i \frac{\varphi}{2}}\right)\right)\right\}=-|L|^{2}(\log (1-\bar{\zeta} z))
\end{gathered}
$$

which completes the proof.

The next theorem is not a simple consequence of the previous one because the class $S_{a \rho \varphi}$ is not compact.

TheOREM 5. If $\lambda \in \mathbb{R}-\{0\}$ then every $f \in S_{a \rho \varphi}$ satisfies the inequality

$$
\begin{gathered}
\operatorname{Re}\left\{\lambda^{2} \log \frac{f^{\prime}(0)}{a-b}+2 \lambda L\left(\log \frac{f(z)-a}{z(f(z)-b)}\right)+L^{2}\left(\log \frac{f(z)-f(\zeta)}{z-\zeta}\right)-\right. \\
\left.-|L|^{2}\left(\log \left(f(z)-e^{i \varphi} \overline{f(\zeta)}-i \rho e^{i \frac{\varphi}{2}}\right)\right)\right\} \leq-|L|^{2}(\log (1-\bar{\zeta} z))
\end{gathered}
$$

The equality occurs for some function $g \in S_{a \rho \varphi}$.

Pr o of. We shall prove that there exists a maximal function $f \in S_{a \rho \varphi}$ for the functional $\psi$ given by the formula (13). This functional is continuous. It is also bounded from above. It follows from the fact that $\left|f^{\prime}(0)\right|$ is bounded, and $\frac{f-a}{f^{\prime}(0)} \in S$ if $f \in S_{a \rho \varphi}$ (S - the class of all functions analytic and univalent in $U$ with normalisation $f(0)=f^{\prime}(0)-1=0$ ), from Growth theorem, from the estimation

$$
\operatorname{Re}\left\{L^{2}\left(\log \frac{g(z)-g(\zeta)}{z-\zeta}\right)\right\} \leq-|L|^{2}(\log (1-\bar{\zeta} z)), \text { for } g \in S[2, \text { p. 116], }
$$

and from the integral representation of the functional from $H^{\prime}(U)$. Suppose that $\lambda \neq 0$. The class $S_{a \rho \varphi}$ is a normal family. Using the fact that $\frac{f-a}{f^{\prime}(0)} \in S$ if $f \in S_{a \rho \varphi}$ we can in a similar manner as in [4] prove that the functional (13) attains its maximal value at some $f \in S_{a \rho \varphi}$.

In the case $\lambda=0$ the inequality (22) also holds but we do not know if there exists in $S_{a \rho \varphi}$ a function for which occurs the equality. However we can prove that this result cannot be improved. 
THEOREM 6. Each function $f \in S_{a \rho \varphi}$ satisfies the inequality

$$
\begin{gathered}
\operatorname{Re}\left\{L^{2}\left(\log \frac{f(z)-f(\zeta)}{z-\zeta}\right)-|L|^{2}\left(\log \left(f(z)-e^{i \varphi} \overline{f(\zeta)}-i \rho e^{i \frac{\varphi}{2}}\right)\right)\right\} \leq \\
\leq-|L|^{2}(\log (1-\bar{\zeta} z))
\end{gathered}
$$

This inequality cannot be improved.

Proof. Applying to (24) the following facts:

(i) there exists a function $\hat{f} \in S$ for which in (23) occurs equality,

(ii) each function from the class $S$ can be approximated by bounded functions from $S$,

(iii) if $g \in S$ is a bounded function then for sufficiently small $r>0$ the function $a+r g \in$ $S_{a \rho \varphi}$

it is easy to see that the left-hand side of (24) can be arbitrarily near the right-hand side, so this result is best possible.

5. Examples. To illustrate the theorems given above, consider two special functionals from $H^{\prime}(U)$. At first let the functional $L$ have the form

$$
L(g)=\sum_{m=1}^{N} \lambda_{m}\left[g\left(z_{m}\right)-g(0)\right], \text { where } g \in H(U), z_{1}, \ldots, z_{N} \in U, \lambda_{1}, \ldots, \lambda_{N} \in \mathbb{C} .
$$

Then (22) leads to the following inequality :

$$
\begin{gathered}
\operatorname{Re}\left\{\left(\lambda-\sum_{m=1}^{N} \lambda_{m}\right)^{2} \log \frac{f^{\prime}(0)}{a-b}+2 \lambda \sum_{m=1}^{N} \lambda_{m} \log \frac{f\left(z_{m}\right)-a}{z_{m}\left(f\left(z_{m}\right)-b\right)}+\right. \\
+\sum_{n, m=1}^{N} \lambda_{n} \lambda_{m} \log \frac{f\left(z_{m}\right)-f\left(z_{n}\right)}{z_{m}-z_{n}} \frac{z_{n} z_{m}(a-b)}{\left(f\left(z_{n}\right)-a\right)\left(f\left(z_{m}\right)-a\right)}- \\
\left.-\sum_{n, m=1}^{N} \lambda_{n} \bar{\lambda}_{m} \log \frac{f\left(z_{n}\right)-e^{i \varphi} \overline{f\left(z_{m}\right)}-i \rho e^{i \frac{\varphi}{2}}}{a-e^{i \varphi} \overline{f\left(z_{m}\right)}-i \rho e^{i \frac{\varphi}{2}}} \cdot \frac{a-b}{f\left(z_{n}\right)-b}\right\} \leq \\
\leq-\sum_{n, m=1}^{N} \lambda_{n} \bar{\lambda}_{m} \log \left(1-z_{n} \bar{z}_{m}\right),
\end{gathered}
$$

where for $\frac{f\left(z_{m}\right)-f\left(z_{n}\right)}{z_{m}-z_{n}}$ we take $f^{\prime}\left(z_{m}\right)$ in the case $n=m$.

Putting $N=1, \lambda=\lambda_{1}=1, z_{1}=z$ in the above inequality we obtain the following estimation:

$$
\frac{\left|f^{\prime}(z)\right|}{\left|f(z)-e^{i \varphi} \overline{f(z)}-i \rho e^{i \frac{\varphi}{2}}\right|} \leq \frac{1}{1-|z|^{2}}
$$

and for $z=0$ we have

$$
\left|f^{\prime}(0)\right| \leq|a-b|
$$

Considering the functional

$$
L(g)=\sum_{m=1}^{N} \lambda_{m} g^{\prime}\left(z_{m}\right), \text { where } g \in H(U), \quad z_{1}, \ldots, z_{N} \in U, \lambda_{1}, \ldots, \lambda_{N} \in \mathbb{C} .
$$


and applying it to the inequality (22) we get

$$
\begin{gathered}
\operatorname{Re}\left\{\lambda^{2} \log \frac{f^{\prime}(0)}{a-b}+2 \lambda \sum_{m=1}^{N} \lambda_{m}\left(\frac{(a-b) f^{\prime}\left(z_{m}\right)}{\left(f\left(z_{m}\right)-a\right)\left(f\left(z_{m}\right)-b\right)}-\frac{1}{z_{m}}\right)+\right. \\
+\sum_{n, m=1}^{N} \lambda_{n} \lambda_{m}\left(\frac{f^{\prime}\left(z_{m}\right) f^{\prime}\left(z_{n}\right)}{\left(f\left(z_{m}\right)-f\left(z_{n}\right)\right)^{2}}-\frac{1}{\left(z_{m}-z_{n}\right)^{2}}\right)- \\
\left.-\sum_{n, m=1}^{N} \lambda_{n} \bar{\lambda}_{m} \frac{e^{i \varphi} \overline{f^{\prime}\left(z_{m}\right)} f^{\prime}\left(z_{n}\right)}{\left(f\left(z_{n}\right)-e^{i \varphi} \overline{f\left(z_{m}\right)}-i \rho e^{i \frac{\varphi}{2}}\right)^{2}}\right\} \leq \\
\leq \sum_{n, m=1}^{N} \lambda_{n} \bar{\lambda}_{m} \frac{1}{\left(1-z_{n} \overline{z_{m}}\right)^{2}} .
\end{gathered}
$$

Because $\lim _{n \rightarrow m} \frac{f^{\prime}\left(z_{m}\right) f^{\prime}\left(z_{n}\right)}{\left(f\left(z_{m}\right)-f\left(z_{n}\right)\right)^{2}}=\frac{1}{6}\left\{f\left(z_{m}\right), z_{m}\right\}$, where $\left\{f\left(z_{m}\right), z_{m}\right\}$ denotes the Schwarzian derivative, then in the case $N=1, z_{1}=z$ we have

$$
\begin{gathered}
\operatorname{Re}\left\{\lambda^{2} \log \frac{f^{\prime}(0)}{a-b}+2 \lambda \lambda_{1}\left(\frac{(a-b) f^{\prime}(z)}{(f(z)-a)(f(z)-b)}-\frac{1}{z}\right)+\frac{1}{6} \lambda_{1}^{2}\{f(z), z\}-\right. \\
\left.-\left|\lambda_{1}\right|^{2} \frac{e^{i \varphi}\left|f^{\prime}(z)\right|^{2}}{\left(f(z)-e^{i \varphi} \overline{f(z)}-i \rho e^{i \frac{\varphi}{2}}\right)^{2}}\right\} \leq\left|\lambda_{1}\right|^{2} \frac{1}{\left(1-|z|^{2}\right)^{2}} .
\end{gathered}
$$

For $\lambda=0$ we get the following estimation:

$$
|\{f(z), z\}| \leq \frac{6}{\left(1-|z|^{2}\right)^{2}}-\frac{6\left|f^{\prime}(z)\right|^{2}}{\left|f(z)-e^{i \varphi} \overline{f(z)}-i \rho e^{i \frac{\varphi}{2}}\right|^{2}} .
$$

\section{References}

[1] R. Caccioppoli, Sui funzionali lineari nel campo delle funzioni analitiche, Atti Accad. Naz. Lincei Rend. Cl. Sci. Fis. Mat. Natur. 13 (1931), 263-266.

[2] G. M. Goluzin, Geometričeskaya teorya funkcii kompleksnogo peremennogo, Moskwa 1966, 68-109, 157-158.

[3] H. Jondro, Sur une méthode variationnelle dans la famille des fonctions de Grunsky-Shah, Bull. Acad. Polon. Sci. 27 (1979), 541-547.

[4] H. Jondro, Les inégalités du type de Grunsky pour les fonctions de la classe K, Ann. Polon. Math. 45 (1985), 43-53.

[5] G. Schober, Univalent functions, Selected Topics, Lecture Notes in Mathematics 478, Springer-Verlag 1975. 